\title{
Usefulness of Bronchoscopic Probe-Based Confocal Laser Endomicroscopy in the Diagnosis of Pneumocystis jirovecii Pneumonia
}

\author{
Hanaa Shafiek ${ }^{a, h}$ Federico Fiorentino ${ }^{a, d}$ Borja G. Cosio ${ }^{a, d, f}$ Ana Kersul \\ Luc Thiberville $^{i}$ Cristina Gómez ${ }^{b}$ Melchor Riera ${ }^{c}$ Maria L. Martín ${ }^{c}$ \\ Rocio Martínez ${ }^{a}$ Maria A. Noguera ${ }^{a}$ Alvar Agustíf,g Jaume Sauleda ${ }^{a}$ d,f

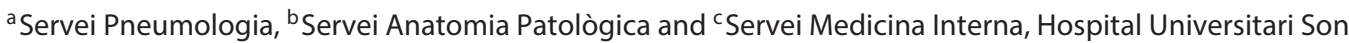 \\ Espases, ${ }^{d}$ Institut d'Investigació Sanitària de Palma (IdISPa), and ${ }^{\mathrm{e}} \mathrm{Cl}$ ínica Palma-Planas, Palma de Mallorca, ${ }^{\mathrm{f}} \mathrm{CIBER}$ \\ de Enfermedades Respiratorias (CIBERES), Madrid, and ${ }^{9}$ Institut del Tórax, Hospital Clínic, IDIBAPS, University of

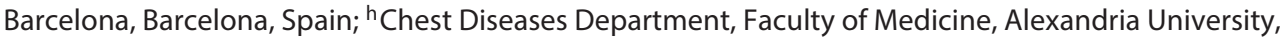 \\ Alexandria, Egypt; iService de Pneumologie, CHU de Rouen, Hôpital Charles Nicolle, Rouen, France
}

\section{Key Words}

Alveoloscopy · Human immunodeficiency virus .

Opportunistic respiratory infections

\begin{abstract}
Background: Probe-based confocal laser endomicroscopy ( $p C L E)$ is a novel technique that provides in vivo microscopic imaging of the distal lung. We hypothesized that the intraalveolar exudates characterizing Pneumocystis jirovecii pneumonia (PJP) can be identified by pCLE in vivo and help in its diagnosis. Objectives: We aimed to assess the usefulness of pCLE for the in vivo diagnosis of PJP. Methods: Thirty-two human immunodeficiency virus (HIV)-positive patients with new pulmonary infiltrates and fever were studied using pCLE. Real-time alveolar images were recorded during the bronchoscopy for off-line analysis by two independent observers. Bronchoalveolar lavage samples were also obtained and processed for microbiology and cytological evaluation, including Grocott stain for $P$. jirovecii. The sensitivity, specificity, positive predictive value (PPV) and negative predictive value (NPV) of $\mathrm{pCLE}$ for the diagnosis of PJP in these patients
\end{abstract}

\section{KARGER}

E-Mail karger@karger.com

www.karger.com/res were calculated. Results: Fourteen patients (44\%) were confirmed to have PJP by cultures/staining. PCLE was well tolerated in all patients. It identified intra-alveolar exudates in 13 of them (41\%), where 11 of them (85\%) had positive Grocott stain for $P$. jirovecci, with $93 \%$ concordance between observers. Sensitivity, specificity, PPV and NPV of pCLE for the diagnosis of PJP were 79, 89, 85 and $84 \%$, respectively. In smokers, these figures improved to be $92,88,85$ and $94 \%$. Conclusions: pCLE is a quick and safe procedure for on-site diagnosis of PJP in HIV+ patients with excellent specificity and sensitivity mainly in smokers.

(c) 2016 S. Karger AG, Basel

\section{Introduction}

Pneumocystis jirovecii pneumonia (PJP) is one of the most prevalent opportunistic infections in patients infected with the human immunodeficiency virus (HIV) [1]. Patients with PJP often complain of subacute symptoms and present with interstitial infiltrates on chest Xray and severe gas exchange impairment [2]. If not diag-
C 2016 S. Karger AG, Basel

0025-7931/16/0921-0040\$39.50/0
Dr. Jaume Sauleda

Servei Pneumologia, Hospital Universitari Son Espases Carretera Valldemossa 79 ES-07010 Palma de Mallorca (Spain)

E-Mail jaume.sauleda@ssib.es 


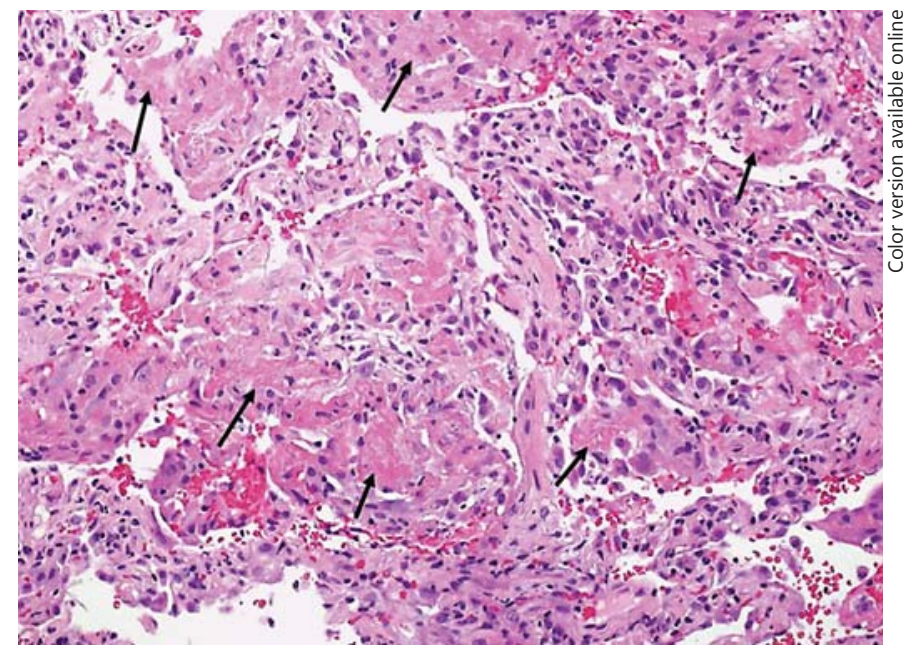

Fig. 1. Characteristic intra-alveolar exudate in PJP (hematoxylineosin) formed by proteins, dead cells and P. jirovecii (arrows). HE. $\times 200$.

nosed and treated promptly, PJP may progress to severe respiratory failure requiring mechanical ventilation and, eventually, death [3]. On-site PJP diagnosis is, therefore, of great clinical relevance [4].

In clinical practice, the diagnosis of PJP may be confounded by the fact that symptoms and chest X-ray findings are similar to those of bacterial pneumonia, also frequent in HIV+ patients. The definitive PJP diagnosis requires the demonstration of $P$. jirovecii in respiratory samples using specific silver staining, like Gomori or Grocott [2] or direct immunofluorescence [5]. However, these techniques are time-consuming and delay PJP diagnosis by $24-72 \mathrm{~h}$, thus delaying the initiation of specific therapy (hence, worsening prognosis) or forcing the start of empiric therapy (with the risk of undesired side effects) [6]. An alternative to these diagnostic techniques, available only in some centers, is the use of molecular techniques to identify $P$. jirovecii DNA in respiratory samples by polymerase chain reaction (PCR) [7]. However, this is an expensive procedure that is also time-consuming. New and more direct diagnostic tools are therefore desirable for the early diagnosis of PJP [4].

Probe-based confocal laser endomicroscopy (pCLE) is a new minimally invasive technique that provides realtime in vivo microscopic imaging of the distal lung [3]. It involves the advancement of a miniprobe through the working channel of the fiberoptic bronchoscope. A laser beam at the end of the miniprobe elicits fluorescence of the tissue surface which is recorded in the system and analyzed off-line [8]. As we have shown recently [9],
pCLE can image and quantify the elastin network of the airways including alveolar ducts, alveolar entrance rings and extra-alveolar microvessels (alveoloscopy), as well as alveolar macrophages in vivo [3].

Considering that PJP is characterized by the presence of intra-alveolar foamy exudates formed by a conglomeration of proteins, dead cells and non-staining $P$. jirovecii (fig. 1) [10,11], and that they are highly sensitive and specific for PJP [12], we hypothesized that pCLE might have the potential to detect these exudates and, therefore, diagnose PJP in vivo, on site. We explored this hypothesis by determining the sensitivity, specificity, positive predictive value (PPV) and negative predictive value (NPV) of pCLE in the diagnosis of PJP in HIV+ patients.

\section{Materials and Methods}

\section{Study Design}

Patients with HIV acutely admitted to our hospital (Hospital Universitari Son Espases, Palma de Mallorca, Spain) with new uni- or bilateral pulmonary infiltrates and fever, from November 2008 to August 2013, were invited to participate. Exclusion criteria were severe acute respiratory failure $\left(\mathrm{PaO}_{2} / \mathrm{FIO}_{2}<250\right)$, coagulation disorder (platelets $<50.000 \mathrm{cell} / \mathrm{mm}^{3}$, prothrombin time test $<50 \%$ ) and/or general contraindications for bronchoscopy according to international guideline recommendations [13]. All participants voluntarily signed their informed consent. The study protocol had been previously approved by the local Ethics Committee.

\section{Patient Characterization}

Complete physical examination, blood cell count, general biochemistry, arterial blood gases and chest X-ray (or computed tomography with intravenous contrast if clinically indicated) were assessed in all patients. Antibiotic therapy started before bronchoscopy (if any) was also recorded.

\section{Bronchoscopy}

Fiberoptic bronchoscope was performed using a Pentax, EB$1570 \mathrm{~K}$ endoscope (Tokyo, Japan). Bronchial aspirate (BAS) and bronchoalveolar lavage (BAL) samples were obtained following international recommendations [14]. Briefly, BAL was performed instilling 100-125 $\mathrm{ml}$ of normal saline in 25-ml aliquots in affected segments (mostly middle lobe and lingula) [14].

\section{Microbiologic Determinations}

Both BAS and BAL samples were processed both for microbiology (Gram and Ziehl-Nielsen stain and bacterial, fungal, viral and mycobacterium cultures) and cytological evaluation (total and differential cell count and Grocott stain for P. jirovecii in BAL samples) and direct immunofluorescence. PCR for PJP was not performed.

\section{Probe-Based Confocal Laser Endomicroscopy}

pCLE (Cellvizio ${ }^{\circledR}$-Lung, $488 \mathrm{~nm}$ excitation, Mauna Kea Technologies, Paris, France) was used, as previously described by Thi- 

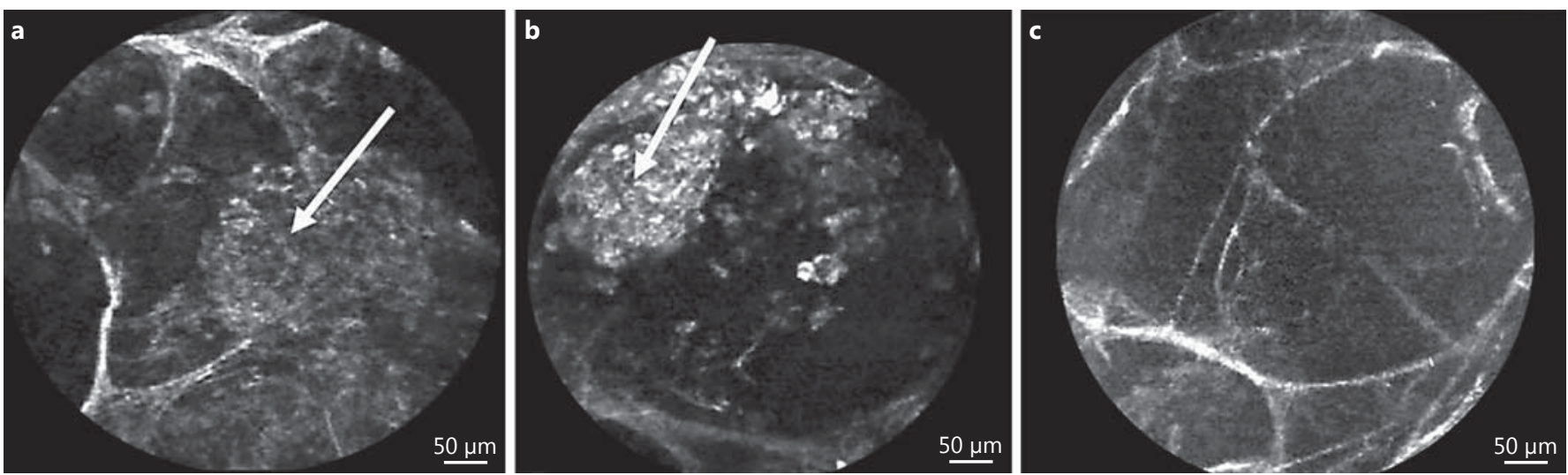

Fig. 2. Intra-alveolar exudates (arrows) detected by pCLE in 2 patients with PJP (a, b). Normal pCLE (c).

Table 1. Characteristics of the 32 patients and the BAL cellular counts

\begin{tabular}{|c|c|c|c|}
\hline & \multicolumn{3}{|l|}{ Presence of $P$. jirovecii } \\
\hline & yes $(n=14 ; 43.8 \%)$ & no $(\mathrm{n}=18 ; 56.2 \%)$ & $\mathrm{p}$ value \\
\hline Age, years & $43.3 \pm 8.3$ & $43 \pm 8.8$ & 0.629 \\
\hline Gender & & & 0.554 \\
\hline Male & $11(78.6)$ & $15(83.3)$ & \\
\hline Female & $3(21.4)$ & $3(16.7)$ & \\
\hline Smoking history & & & 0.24 \\
\hline Smoker & $12(85.7)$ & $17(94.4)$ & \\
\hline Nonsmoker & $2(14.3)$ & $1(5.6)$ & \\
\hline $\mathrm{PaO}_{2}$ & $67.6 \pm 21.9$ & $64.9 \pm 21.5$ & 0.959 \\
\hline $\mathrm{FIO}_{2} \%$ & $42.6 \pm 34.5$ & $26.3 \pm 20.4$ & 0.872 \\
\hline HIV stage $\mathrm{e}^{\mathrm{a}}$ & & & $0.011^{*}$ \\
\hline Stage C & $14(100)$ & $11(61.1)$ & \\
\hline Stage B & 0 & $4(22.2)$ & \\
\hline Stage A & 0 & $3(16.7)$ & \\
\hline Median CD4 count, cells $/ \mathrm{mm}^{3}$ (IQR) & $30(21-50)$ & $159(134.25-255.25)$ & $0.0001^{*}$ \\
\hline Median viral count, copies/ml (IQR) & $148,800(76,483.25-408,752.75)$ & $4,103.5(248.5-168,147.5)$ & $0.016^{*}$ \\
\hline \multicolumn{4}{|l|}{ BAL } \\
\hline Lymphocytes, \% & $17.3 \pm 15$ & $13.5 \pm 8.2$ & 0.829 \\
\hline Macrophages, \% & $64 \pm 21$ & $64.8 \pm 28.6$ & 0.766 \\
\hline PMN, \% & $18.3 \pm 17.8$ & $23 \pm 31$ & 0.733 \\
\hline
\end{tabular}

Figures are mean $\pm \mathrm{SD}$ or numbers with percentages in parentheses. $\mathrm{PMN}=$ Polymorphonuclear neutrophils; FIO $2=$ fraction of inspired oxygen $\mathrm{PaO}_{2}=$ arterial oxygen tension; $\mathrm{IQR}=$ interquartile range (percentile $\left.25-75\right) .{ }^{*} \mathrm{p}<0.05$.

a According to CDC staging.

berville et al. [3] and Cosio et al. [9]. In brief, the bronchoscope is wedged into the smallest reachable bronchi and a Confocal Miniprobe $^{\mathrm{TM}}$ (AlveoFlex, $1.4 \mathrm{~mm}$ in diameter, Paris, France) introduced through its working channel and advanced into the distal bronchiole until the alveolar space is seen. Then, the probe is pulled back slightly to minimize compression [3]. Alveolar images were obtained in 4 different lung segments among those with most radiological abnormalities. Real-time alveolar images were continuously recorded during the procedure and stored for off- line analysis, as detailed below. After bronchoscopy, a plain chest $\mathrm{X}$-ray was obtained routinely. The duration of pCLE procedure was recorded.

\section{Off-Line pCLE Analysis}

We considered that the patient had intra-alveolar exudates if at least one homogeneous intra-alveolar infiltrate was observed offline (fig. 2) by two independent observers. In case of discordance, a final consensus decision was reached after a new analysis of the 
Table 2. Individual microbiologic and pCLE results

\begin{tabular}{|c|c|c|c|c|}
\hline \multirow[t]{2}{*}{$\begin{array}{l}\text { Patient } \\
\text { ID }\end{array}$} & \multirow[t]{2}{*}{ Microbiologic cultures } & \multicolumn{3}{|c|}{$\begin{array}{l}\text { Presence of intra-alveolar } \\
\text { exudates (pCLE) }\end{array}$} \\
\hline & & $\begin{array}{l}\text { two } \\
\text { observers }\end{array}$ & $\begin{array}{l}\text { 1st } \\
\text { observer }\end{array}$ & $\begin{array}{l}\text { 2nd } \\
\text { observer }\end{array}$ \\
\hline 1 & BAS: E. coli & - & - & - \\
\hline 2 & BAS: S. aureus sensitive to methicillin + Candida parasilopsis & - & - & - \\
\hline 3 & BAS and BAL: $P$. jirovecci & + & + & + \\
\hline 4 & BAS and BAL: S. pneumoniae & - & - & - \\
\hline 5 & Negative culture & - & - & - \\
\hline 6 & BAS and BAL: M. tuberculosis and Candida albicans & - & - & - \\
\hline 7 & BAS and BAL: $P$. jirovecci & + & + & + \\
\hline 8 & BAS and BAL: CMV & - & - & - \\
\hline 9 & BAS and BAL: $M$. tuberculosis & + & + & + \\
\hline 10 & BAS and BAL: $M$. tuberculosis & - & - & - \\
\hline 11 & BAS and BAL: $P$. jirovecci & + & + & + \\
\hline 12 & Transbronchial biopsy: Mycobacterium avium-intracellulare & - & - & - \\
\hline 13 & Negative culture & - & - & - \\
\hline 14 & Negative culture & - & - & - \\
\hline 15 & Negative culture & - & - & - \\
\hline 16 & BAS and BAL: K. pneumoniae & - & - & - \\
\hline 17 & BAS and BAL: P. jirovecci & - & - & - \\
\hline 18 & Negative culture & + & + & + \\
\hline 19 & BAS and BAL: P. jirovecci & + & + & + \\
\hline 20 & BAS and BAL: $P$. jirovecci & + & + & + \\
\hline 21 & BAS and BAL: H. influenzae & - & - & - \\
\hline 22 & BAS and BAL: $P$. jirovecci & + & + & + \\
\hline 23 & BAS and BAL: P. jirovecci & + & + & + \\
\hline 24 & BAS and BAL: P. jirovecci & + & + & + \\
\hline 25 & BAS and BAL: P. jirovecci & - & - & + \\
\hline 26 & Negative culture & - & - & - \\
\hline 27 & Negative culture & - & - & - \\
\hline 28 & BAS and BAL: $P$. jirovecci & - & - & - \\
\hline 29 & Negative culture & - & - & - \\
\hline 30 & BAS and BAL: P. jirovecci & + & + & + \\
\hline 31 & BAS and BAL: P. jirovecci & + & + & + \\
\hline 32 & BAS and BAL: P. jirovecci & + & + & + \\
\hline
\end{tabular}

imaging sequence by the two observers together. Build-in software (MedViewer ${ }^{\circledR}$ 1.1.1; Mauna Kea Technologies, Paris, France) was used for the measurement of the extra-alveolar vessel diameter, alveolar diameter, alveolar elastic fiber thickness and alveolar fluorescence intensity as previously described $[3,8,9]$.

\section{Statistical Analysis}

Results are presented as mean \pm standard deviation (SD) or number (percentage) as appropriate. Characteristics of patients with and without PJP were compared using the Mann-Whitney test or $\chi^{2}$ test as appropriate, unless otherwise stated. Concordance between the two pCLE observers was assessed using the kappa index. Receiver operating characteristic (ROC) curve was constructed and the area under the curve (AUC) determined. Sensitivity, specificity, PPV and NPV of pCLE for the diagnosis of PJP in these patients were also calculated. A p value $<0.05$ (two-tailed) was considered significant. Analysis was performed using MedCalc ${ }^{\circledR}$ (version 9.2.1.0, Acacialaan 22, Ostend, Belgium).

\section{Results}

\section{Clinical Characteristics of Participants}

We studied $32 \mathrm{HIV}+$ patients (26 males and 6 females) hospitalized because of acute febrile pulmonary infiltrates. Table 1 presents their main clinical characteristics. 
Fig. 3. a ROC of pCLE to detect intra-alveolar exudates in all $\mathrm{HIV}+$ patients $(\mathrm{AUC}=$ $0.837, \mathrm{p}=0.0001)$. $\mathbf{b}$ ROC of pCLE to detect intra-alveolar exudates in HIV+ patients being smokers $(\mathrm{AUC}=0.900, \mathrm{p}=0.0001)$.

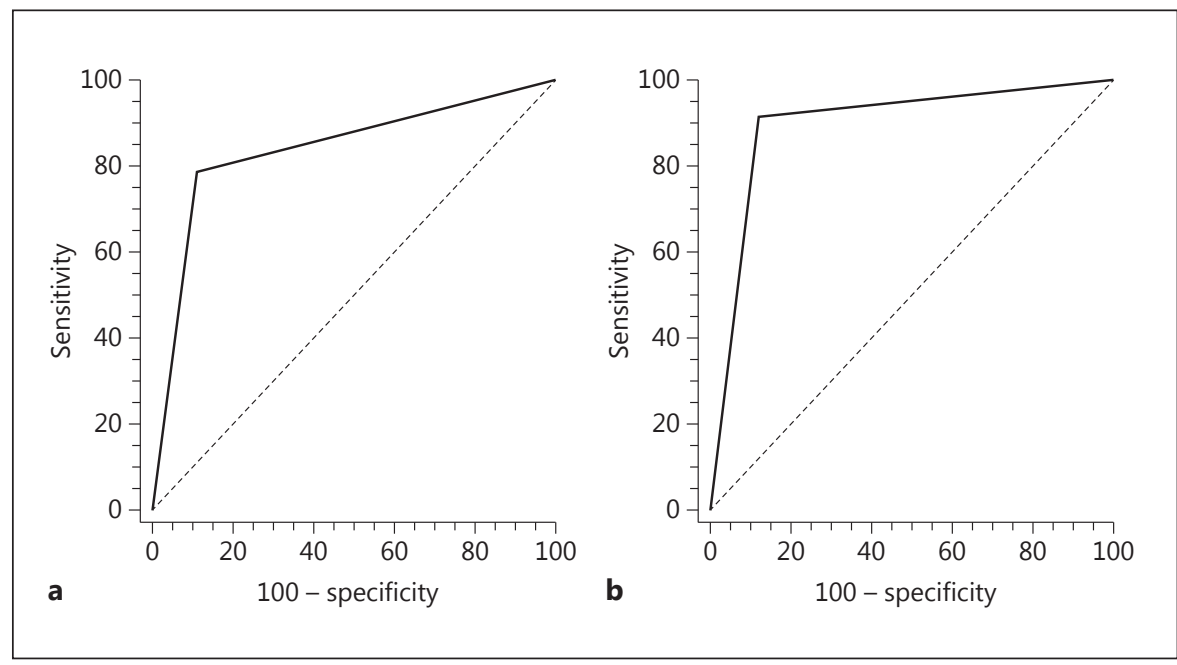

Mean age was 43 years and there was a preponderance of males. Most patients were current smokers. Pulmonary gas exchange was moderately to severely impaired (table 1). As expected, we found a higher HIV stage, lower CD4 lymphocytes and higher HIV copies in patients with PJP $(n=14)$ than in those without $(n=18)($ table 1$)$.

Table 2 shows the microbiological results of BAS and BAL in each individual. Grocott stain was positive for $P$. jirovecii in 14 patients (44\%), cultures/staining were negative in 8 patients (25\%) and other micro-organisms were detected in the remaining 10 patients $(31 \%)$. We found 2 false positives (11\%) in the 18 patients (56\%) without PJP.

\section{Bronchoscopy and pCLE}

Bronchoscopy and pCLE were well tolerated by all participants, and no patient developed any significant complication during or after the procedures. The mean duration of pCLE was $3.9 \pm 2.7 \mathrm{~min}$ ( $\max .13 \mathrm{~min}$ in 1 case). Table 2 also presents pCLE presence/absence of alveolar exudates in each individual. Concordance between the two observers was 93\%. pCLE identified intra-alveolar exudates in 13 patients (41\%) of which 11 (85\%) had positive Grocott stain for $P$. jirovecci. In 3 patients out of the $14(21 \%)$ with positive $P$. jirovecci stains (ID 17, 25 and 28 in table 2), pCLE did not detect intra-alveolar exudates. Two of these 3 patients were nonsmokers, and all of them had started treatment for $P$. jirovecci [sulfamethoxazole/trimethoprim $800 / 160 \mathrm{mg}$ plus corticosteroids (methylprednisolone $40 \mathrm{mg} / 24 \mathrm{~h}$ )] $\geq 48 \mathrm{~h}$ before pCLE. The sensitivity, specificity, PPV and NPV of pCLE for the identification of intra-alveolar exudates in PJP were $79,89,85$ and $84 \%$, respectively $($ AUC $=0.837, p=$
Table 3. The frequency of both positive and negative pCLE and $P$. jirovecci detected microbiologically among the smokers

\begin{tabular}{lccc}
\hline & \multicolumn{2}{c}{ pCLE } & \multirow{2}{*}{ Total } \\
\cline { 2 - 3 } & negative & positive & \\
\hline Presence of $P$. jirovecci & & & \\
No & $15(93.7 \%)$ & $2(15 \%)$ & $17(58.6 \%)$ \\
Yes & $1(6.3 \%)$ & $11(85 \%)$ & $12(41.4 \%)$ \\
\hline Total & $16(55.2 \%)$ & $13(44.8 \%)$ & $29(100 \%)$ \\
\hline Fisher's exact test (p value) & & & $<0.0001^{*}$ \\
\hline
\end{tabular}

0.0001; fig. 3a). In smokers only $(\mathrm{n}=29,91 \%)$, these figures were $92,88,85$ and $94 \%$, respectively $(A U C=0.9$, $\mathrm{p}=0.0001$; fig. $3 \mathrm{~b}$ ). Table 3 shows the frequencies of positive and negative pCLE detection of intra-alveolar exudates among the smokers group.

Tables 4 and 5 show several morphometric measurements obtained using pCLE regarding smoking history or pneumocystis infection, respectively. There were no significant differences except for the alveolar diameter that was lower in patients with PJP (table 5).

\section{Discussion}

The results of this study indicate that pCLE is a safe and efficacious procedure for real-time, on-site, in vivo diagnosis of PJP in HIV+ patients. 
Table 4. Morphometric measurements

\begin{tabular}{lccc}
\hline Morphometric measurements & $\begin{array}{l}\text { Smokers } \\
(\mathrm{n}=29)\end{array}$ & $\begin{array}{l}\text { Nonsmokers } \\
(\mathrm{n}=3)\end{array}$ & $\mathrm{p}$ value \\
\hline Alveolar diameter, $\mu \mathrm{m}$ & $257.97 \pm 33.3$ & $239 \pm 65.8$ & 0.539 \\
Alveolar elastic fiber diameter, $\mu \mathrm{m}$ & $13.4 \pm 2.4$ & $12.4 \pm 0.9$ & 0.348 \\
Extra-alveolar vessel diameter, $\mu \mathrm{m}$ & $131.04 \pm 43.1$ & $135.03 \pm 49.3$ & 0.841 \\
Alveolar intensity, $\mu \mathrm{m}$ & $216.8 \pm 115.2$ & $145.6 \pm 121.4$ & 0.349 \\
Macrophages, $\mathrm{n}(\%)$ & $27(84.4)$ & $2(6.2)$ & 0.263 \\
\hline
\end{tabular}

Table 5. Morphometric measurements among the studied cohort of patients

\begin{tabular}{lccc}
\hline Morphometric measurements & \multicolumn{2}{l}{ Presence of $P$. jirovecii } \\
\cline { 2 - 4 } & yes $(\mathrm{n}=14)$ & no $(\mathrm{n}=18)$ & $\mathrm{p}$ value \\
\hline Alveolar diameter, $\mu \mathrm{m}$ & $236.6 \pm 39.8$ & $271.5 \pm 24.9$ & $0.028^{*}$ \\
Alveolar elastic fiber diameter, $\mu \mathrm{m}$ & $12.4 \pm 1.9$ & $14 \pm 2.4$ & 0.074 \\
Extra-alveolar vessel diameter, $\mu \mathrm{m}$ & $131.7 \pm 54.7$ & $131.2 \pm 33.7$ & 0.575 \\
Alveolar intensity & $188.3 \pm 142.4$ & $227.1 \pm 90.6$ & 0.224 \\
Macrophages, $\mathrm{n}(\%)$ & $12(37.5)$ & $17(53.13)$ & 0.408 \\
\hline \multirow{2}{*}{$* \mathrm{p}<0.05}$. & & & \\
\hline
\end{tabular}

\section{Previous Studies}

Previous studies used pCLE to investigate interstitial lung disease, alveolar proteinosis, drug-induced (amiodarone) pneumonitis, lung cancer [15-18] and, very recently, chronic obstructive pulmonary disease [9]. To the best of our knowledge, this is the first study to investigate the potential clinical usefulness of pCLE in the diagnosis of PJP in HIV+ patients.

\section{Safety of the Procedure}

Previous studies have reported a minor risk of pneumothorax and chest pain [15] or mild bleeding [19] following pCLE. This was not the case in any of the patients included in our study. Of note, however, we excluded patients with severe acute respiratory failure and mechanical ventilation. Therefore, our results cannot be extended directly to more severely ill HIV+ patients.

\section{Usefulness of $p C L E$ for the Diagnosis of PJP}

Despite the availability of highly active antiretroviral therapy and the widespread use of PJP prophylaxis [20], the latter still is one of the commonest opportunistic infections in HIV+ patients [21, 22]. Additionally, the high mortality of patients with PJP requiring mechanical ventilation remains unchanged (50-60\%) [23]. To improve survival, early empirical treatment with trimethoprim/ sulfamethoxazole is generally recommended [24]. Yet, this can have severe adverse effects which are considered high in HIV+ patients ranging between 20 and 85\% [6, 25]. Importantly, there is no currently available rapid test for the on-site accurate diagnosis of PJP. The classical diagnostic method (Gomori, Grocott silver staining) usually requires $48 \mathrm{~h}$. Immunofluorescence and molecular tests such as PCR are not available in all centers and final results also need several hours or days.

Our study shows that pCLE allows the identification of alveolar exudates that closely resemble those found in lung biopsies (fig. 2) and that its presence is highly specific for PJP (89\%), with a sensitivity of 79\%, a PPV of $85 \%$ and an NPV of $84 \%$. Several factors can contribute to explain the absence of pCLE exudates in 3 patients with PJP (false negatives). First, they had started empiric antibiotic treatment 3-5 days before pCLE; this can improve the clinical condition of mild to moderate PJP cases [23] and decrease the diagnostic yield of other methods, such as PCR $[7,26]$. Second, these 3 false-negative patients also received treatment with corticosteroids, known to accelerate the resolution of PJP, particularly if initiated during the first $72 \mathrm{~h}$ of hospitalization [27-29]. Third, interestingly, 2 of these 3 patients were nonsmokers. Whether or not smoking facilitates the formation of alveolar exudates in PJP patients is unknown. Yet, it is well established that the number of alveolar macrophages (an integral component of these exudates) is higher in smokers than nonsmokers [30], and that smoking increases their autofluorescence [8] and surfactant protein A both in plasma and 
sputum [31]. The latter is potentially relevant in the context of this study since Salaün et al. [16] and Koziel et al. [32] reported that $P$. jirovecii infection was also associated with increased levels of surfactant protein A in the alveoli, which is part of the proteinaceous material of intraalveolar exudates [11]. Thus, the combination of these factors can contribute to explain a better diagnostic performance of pCLE in smokers (sensitivity $92 \%$, specificity $88 \%$, PPV $85 \%$ and NPV $94 \%$ ).

We found no morphometric differences between smokers and nonsmokers (table 3 ) and lower alveolar diameter in patients with PJP (table 4). The latter could be due to some degree of fibrosis that has been reported in lung biopsies in these patients [33].

\section{Clinical Implications}

All in all, our observations indicate that pCLE offers the possibility of a rapid on-site diagnosis and consequently that treatment can be initiated quickly based on a specific diagnosis that may improve prognosis. Likewise, we show that pCLE can also contribute to exclude this diagnosis, thus forcing the clinician to consider the presence of other microbiologic agents, such as bacterial or mycobacterial infection that require a different therapeutic strategy. There are other lesser invasive methods than bronchoscopy that has a good yield of $90 \%$ such as oral washings with PCR-based diagnostics or detection of blood $\beta$-glucan $[34,35]$. However they are not fully available in all hospitals and $\beta$-glucan cannot rule out other microorganisms, especially fungal infection [36] as well as a risk of detecting subclinical colonization by $P$. jiroveci using PCR [37]. Bronchoscopy is a minimally invasive procedure that allows pCLE but can also obtain respiratory secretions and bronchoalveolar lavage allowing their analyses identifying other potential microorganisms. We do not defend the replacement of lesser invasive techniques but we stress a new inclusion of pCLE indication besides pulmonary aspergillosis, alveolar proteinosis, drug pneumonitis and lung cancer [15-18, 38, 39].

\section{Strengths and Limitations}

The present study is the first to report the usefulness of pCLE in the diagnosis of PJP but has some limitations that deserve comment. First, practically, all parts of the lung cannot be imaged by confocal endomiscroscopy; thus, alveolar exudates and PJP can be missed in some cases. However, according to the study protocol, we imaged different lung segments with most radiological abnormalities. Second, the present study includes a relatively small number of patients. This is likely to be a fortunate conse-

quence of the new antiretroviral treatments that reduces drastically the incidence of opportunistic infections in HIV patients [40]. Yet, further studies in larger cohorts are required to firmly establish the diagnostic usefulness of pCLE in this context. Third, as discussed above, our results cannot be directly extended to more severe patients under mechanical ventilation. Lastly, the present study did not include HIV- patients infected with PJP. However, PJP is more common in HIV+ rather than non-HIV immunocompromised patients owing to various factors including lack of chemoprophylaxis in these patients as well as a high percentage of patients were newly diagnosed with HIV at the time of PJP infection. Further, P. jirovecii prophylaxis in non-HIV immunocompromised patients appears to be more effective than in HIV infection that is associated with a decreased absolute number of PJP cases with an estimated risk reduction of $91 \%$ as being reported by Green et al. [41] and Rodriguez et al. [42].

\section{Conclusions}

This study shows that pCLE is a quick and safe procedure for the on-site diagnosis of PJP in HIV+ patients by providing in vivo 'histological' images of the characteristic intra-alveolar exudates, with excellent specificity and sensitivity, particularly in active smokers.

\section{Acknowledgements}

The authors thank the participants for their willingness to contribute to the study funded by Govern Illes Balears Accions Especials 2009, Premi Son Espases 2008-2009, Direcció General d'Investigació i desenvolupament tecnològic de la Conselleria d'Innovació, Interior i Justícia de la Comunitat Autònoma de les Illes Balears and Fondos FEDER (Grupos competitivos PRE-R-22528-2011).

References

References

infection. Respirology 2008;13:181-190.
2 Luks AM, Neff MJ: Pneumocystis jiroveci pneumonia. Respir Care 2007;52:59-63.

-3 Thiberville L, Salaün M, Lachkar S, Dominique S, Moreno-Swirc S, Vever-Bizet C, et al: Human in vivo fluorescence microimaging of the alveolar ducts and sacs during bronchoscopy. Eur Respir J 2009;33:974-985.

-4 Sauleda J, Broquetas JM, Gea J, Aran X, Conangla M, Gimferrer E, et al: Prognostic implications of bronchoalveolar lavage neutrophilia in patients with Pneumocystis carinii pneumonia and AIDS: effects of an aggressive therapeutic approach. Am Rev Respir Dis 1990;141(4 Pt 1):1080-1081.
Shafiek et al. 
5 Rigole P, Basset D, Dedet JP: Biological diagnosis of pneumocystis infection. Evaluation and value of a new direct immunofluorescent technique. Pathol Biol (Paris) 1997;45:19-23.

-6 Porras MC, Lecumberri JN, Castrillon JL: Trimethoprim/sulfamethoxazole and metabolic acidosis in HIV-infected patients. Ann Pharmacother 1998;32:185-189.

7 Boondireke S, Mungthin M, Tan-ariya P, Boonyongsunchai $P$, Naaglor T, Wattanathum A, et al: Evaluation of sensitivity of multiplex PCR for detection of Mycobacterium tuberculosis and Pneumocystis jirovecii in clinical samples. J Clin Microbiol 2010;48:3165-3168.

$>8$ Thiberville L, Moreno-Swirc S, Vercauteren T, Peltier E, Cave C, Bourg Heckly G: In vivo imaging of the bronchial wall microstructure using fibered confocal fluorescence microscopy. Am J Respir Crit Care Med 2007;175:2231.

-9 Cosio BG, Shafiek H, Fiorentino F, Gomez C, Lopez M, Rios A, et al: Structure-function relationship in COPD revisited: an in vivo microscopy view. Thorax 2014;69:724-730.

10 Travis WD: Surgical pathology of pulmonary infections. Semin Thorac Cardiovasc Surg 1995;7:62-69.

11 Miller RF, Mitchell DM: AIDS and the lung: update 1995. 1. Pneumocystis carinii pneumonia. Thorax 1995;50:191-200.

-12 Tregnago R, Xavier RG, Pereira RP, Prolla JC: The diagnosis of Pneumocystis carinii pneumonia by cytologic evaluation of Papanicolaou and Leishman-stained bronchoalveolar specimens in patients with the acquired immunodeficiency syndrome. Cytopathology 1993;4:77-84.

-13 Guidelines for fiberoptic bronchoscopy in adults. American Thoracic Society. Medical Section of the American Lung Association. Am Rev Respir Dis 1987; 136:1066.

14 Clinical guidelines and indications for bronchoalveolar lavage (BAL): report of the European Society of Pneumology Task Group on BAL. Eur Respir J 1990;3:937-976.

-15 Salaün M, Roussel F, Bourg-Heckly G, VeverBizet C, Dominique S, Genevois A, et al: In vivo probe-based confocal laser endomicroscopy in amiodarone-related pneumonia. Eur Respir J 2013;42:1646-1658.

-16 Salaün M, Roussel F, Hauss PA, Lachkar S, Thiberville L: In vivo imaging of pulmonary alveolar proteinosis using confocal endomicroscopy. Eur Respir J 2010;36:451-453.

17 Fuchs FS, Zirlik S, Hildner K, Schubert J, Vieth M, Neurath MF: Confocal laser endomicroscopy for diagnosing lung cancer in vivo. Eur Respir J 2013;41:1401-1408.

18 Thiberville L, Salaün M, Moreno-Swirc S, Bourg Heckly G: Alveoscopy in diffuse interstitial lung disease. Eur Respir J 2007;712S.

19 Anantham D: Confocal imaging; in Ernst A, Herth FJ (eds): Principles and Practice of Interventional Pulmonology, ed 14. New York, Springer, 2013, pp 227-235.

20 Samperiz G, Guerrero D, Lopez M, Valera J, Iglesias A, Rios A, et al: Prevalence of and risk factors for pulmonary abnormalities in HIVinfected patients treated with antiretroviral therapy. HIV Med 2014;15:321-329.

21 Gebo KA, Diener-West M, Moore RD: Hospitalization rates in an urban cohort after the introduction of highly active antiretroviral therapy. J Acquir Immune Defic Syndr 2001;27: 143-152.

22 Segal R, Poznansky MC, Connors L, Sands K, Barlam T: Changing patterns of presentations of patients with HIV-related disease at a tertiary referral centre and its implications for physician training. Int J STD AIDS 2001;12: 453-459.

23 Mansharamani NG, Garland R, Delaney D, Koziel H: Management and outcome patterns for adult Pneumocystis carinii pneumonia, 1985 to 1995: comparison of HIV-associated cases to other immunocompromised states. Chest 2000;118:704-711.

24 Sauleda J, Gea J, Aran X, Gimferrer E, Conangla M, Broquetas JM: Neutrophilia in the bronchoalveolar lavage of patients with AIDS and Pneumocystis carinii pneumonia. Reflections on its prognostic value in the Spanish setting. Arch Bronconeumol 1994;30:192-195.

25 Kaplan JE, Benson C, Holmes KH, Brooks JT, Pau A, Masur H: Guidelines for prevention and treatment of opportunistic infections in HIV-infected adults and adolescents: recommendations from CDC, the National Institutes of Health, and the HIV Medicine Association of the Infectious Diseases Society of America. MMWR Recomm Rep 2009;58(RR4):1-207; quiz CE1-4.

26 Robberts FJ, Liebowitz LD, Chalkley LJ: Polymerase chain reaction detection of Pneumocystis jiroveci: evaluation of 9 assays. Diagn Microbiol Infect Dis 2007;58:385-392.

27 Bozzette SA, Sattler FR, Chiu J, Wu AW, Gluckstein D, Kemper C, et al: A controlled trial of early adjunctive treatment with corticosteroids for Pneumocystis carinii pneumonia in the acquired immunodeficiency syndrome. California Collaborative Treatment Group. N Engl J Med 1990;323:1451-1457.

28 Walmsley S, Levinton C, Brunton J, Muradali D, Rappaport D, Bast M, et al: A multicenter randomized double-blind placebo-controlled trial of adjunctive corticosteroids in the treatment of Pneumocystis carinii pneumonia complicating the acquired immune deficiency syndrome. J Acquir Immune Defic Syndr Hum Retrovirol 1995;8:348-357.

29 Nielsen TL, Eeftinck Schattenkerk JK, Jensen $B N$, Lundgren JD, Gerstoft J, van Steenwijk RP, et al: Adjunctive corticosteroid therapy for Pneumocystis carinii pneumonia in AIDS: a randomized European multicenter open label study. J Acquir Immune Defic Syndr 1992;5: 726-731.

30 Wallace WA, Gillooly M, Lamb D: Intra-alveolar macrophage numbers in current smokers and non-smokers: a morphometric study of tissue sections. Thorax 1992;47:437-440.

31 Mazur W, Toljamo T, Ohlmeier S, Vuopala K, Nieminen P, Kobayashi H, et al: Elevation of surfactant protein A in plasma and sputum in cigarette smokers. Eur Respir J 2011;38:277284.

32 Koziel H, Phelps DS, Fishman JA, Armstrong MY, Richards FF, Rose RM: Surfactant protein-A reduces binding and phagocytosis of Pneumocystis carinii by human alveolar macrophages in vitro. Am J Respir Cell Mol Biol 1998;18:834-843.

33 Travis WD, Pittaluga S, Lipschik GY, Ognibene FP, Suffredini AF, Masur H, et al: Atypical pathologic manifestations of Pneumocystis carinii pneumonia in the acquired immune deficiency syndrome. Review of 123 lung biopsies from 76 patients with emphasis on cysts, vascular invasion, vasculitis, and granulomas. Am J Surg Pathol 1990;14:615625.

34 Durand-Joly I, Chabe M, Soula F, Delhaes L, Camus D, Dei-Cas E: Molecular diagnosis of Pneumocystis pneumonia. FEMS Immunol Med Microbiol 2005;45:405-410.

35 Wood BR, Komarow L, Zolopa AR, Finkelman MA, Powderly WG, Sax PE: Test performance of blood beta-glucan for Pneumocystis jirovecii pneumonia in patients with AIDS and respiratory symptoms. AIDS 2013;27:967972.

36 He S, Hang JP, Zhang L, Wang F, Zhang DC, Gong FH: A systematic review and meta-analysis of diagnostic accuracy of serum 1,3-beta-D-glucan for invasive fungal infection: focus on cutoff levels. J Microbiol Immunol Infect 2015;48:351-361.

37 Jarboui MA, Sellami A, Sellami H, Cheikhrouhou F, Makni F, Ben Arab N, et al: Molecular diagnosis of Pneumocystis jiroveci pneumonia in immunocompromised patients. Mycoses 2010;53:329-333.

38 Danilevskaya O, Averyanov A, Klimko N, Lesnyak V, Sorokina A, Sazonov D, et al: The case of diagnostics of invasive pulmonary aspergillosis by in vivo probe-based confocal laser endomicroscopy of central and distal airways. Med Mycol Case Rep 2014;5:35-39.

39 Danilevskaya O, Averyanov A, Lesnyak V, Chernyaev A, Sorokina A: Confocal laser endomicroscopy for diagnosis and monitoring of pulmonary alveolar proteinosis. J Bronchology Interv Pulmonol 2015;22:33-40.

40 Kaplan JE, Hanson D, Dworkin MS, Frederick T, Bertolli J, Lindegren ML, et al: Epidemiology of human immunodeficiency virus-associated opportunistic infections in the United States in the era of highly active antiretroviral therapy. Clin Infect Dis 2000;30(suppl 1):S5S14.

41 Green H, Paul M, Vidal L, Leibovici L: Prophylaxis for Pneumocystis pneumonia (PCP) in non-HIV immunocompromised patients. Cochrane Database Syst Rev 2007; 18:CD005590.

42 Rodriguez M, Fishman JA: Prevention of infection due to Pneumocystis spp in human immunodeficiency virus-negative immunocompromised patients. Clin Microbiol Rev 2004; $17: 770-782$.
pCLE in Pneumocystis jirovecii Pneumonia
Respiration 2016;92:40-47 DOI: $10.1159 / 000447431$ 